\title{
Thermo-compositional evolution of the primitive mantle with magma oceans
}

\author{
A. MORISON ${ }^{1}$, D. BOLRÃO ${ }^{2}$, S. LABROSSE ${ }^{1}$, \\ R. Agrusta ${ }^{1}$, A. Rozel ${ }^{2}$, M. BALLMER ${ }^{2,3}$, \\ R. DEGUEN ${ }^{4}$, T. AlboussiÈre ${ }^{1}$, P. TACKLEY ${ }^{2}$
}

${ }^{1}$ ENS de Lyon, UCBL, Univ. de Lyon, France.

adrien.morison@gmail.com

${ }^{2}$ Inst. Of Geophysics, ETH Zürich, Switzerland.

${ }^{3}$ Dept. Earth Sciences, Univ. College London, UK.

${ }^{4}$ ISTerre, Univ. Grenoble-Alpes, France.

Accretional energy and short-lived-elements radioactive heating involved in planets formation is thought to be sufficient to melt the primitive mantle entirely, resulting in a global magma ocean. We consider here the general scenario where the crystallization of such an ocean can happen from the middle or the bottom of the mantle. This leads to a situation where the solid mantle is either surrounded by two global magma oceans, or merely a top magma ocean.

We propose here to study the dynamic and thermocompositional evolution of the primitive solid mantle surrounded by magma oceans and connect them to geochmical and seismic observations. Indeed, the boundaries between the solid and the magma oceans are phase-change interfaces, which has numerous dynamical and chemical consequences. Dynamical exchange of matter can occur between the solid mantle and the magma oceans via melting and freezing. The associated fractional crystallization leads to compositional heterogeneities in the mantle. We solve numerically the equations of solid-state convection in the solid part of the mantle. This model is coupled to $1 \mathrm{D}$ models of crystallization of the magma oceans to self-consistently compute the thickening of the solid part as heat is evacuated from the mantle; as well as the associated fractional crystallization.

We find that the presence of magma oceans greatly affects the convection patterns in the solid as well as its heat flux. Larger-scale convection patterns are selected compared to the classical case with non-penetrative boundary conditions; and the heat transfert in the solid is more efficient. This affects the long term thermal evolution of the mantle as well as the shape of chemical heterogeneities that can be built by fractional crystallization of magma oceans.

Large scale convection patterns produce large LLSVP-like piles at the base of the mantle that constitute a reservoir of incompatible elements. 